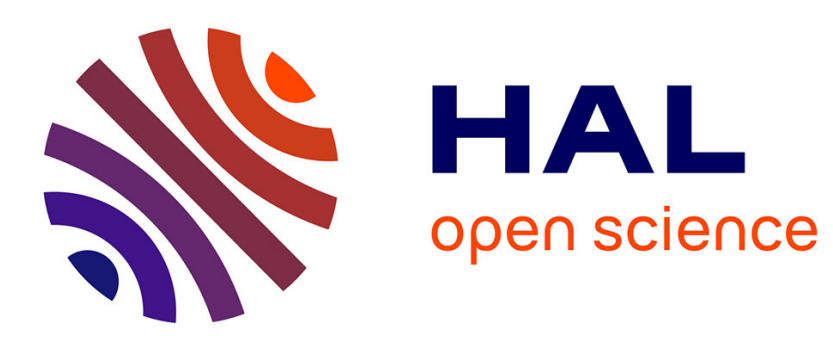

\title{
Vietoris-Rips Complexes also Provide Topologically Correct Reconstructions of Sampled Shapes
}

Dominique Attali, André Lieutier, David Salinas

\section{To cite this version:}

Dominique Attali, André Lieutier, David Salinas. Vietoris-Rips Complexes also Provide Topologically Correct Reconstructions of Sampled Shapes. SoCG 2011 - 27th Annual Symposium on Computational Geometry, Jun 2011, Paris, France. pp.s/n. hal-00579864

\section{HAL Id: hal-00579864 https://hal.science/hal-00579864}

Submitted on 25 Mar 2011

HAL is a multi-disciplinary open access archive for the deposit and dissemination of scientific research documents, whether they are published or not. The documents may come from teaching and research institutions in France or abroad, or from public or private research centers.
L'archive ouverte pluridisciplinaire HAL, est destinée au dépôt et à la diffusion de documents scientifiques de niveau recherche, publiés ou non, émanant des établissements d'enseignement et de recherche français ou étrangers, des laboratoires publics ou privés. 


\title{
Vietoris-Rips Complexes also Provide Topologically Correct Reconstructions of Sampled Shapes*
}

\author{
[Extended Abstract]
}

\author{
Dominique Attali \\ Gipsa-lab \\ Grenoble, France \\ dominique.attali@gipsa- \\ lab.grenoble-inp.fr
}

\author{
André Lieutier \\ Dassault Système \\ Aix-en-Provence, France \\ andre.lieutier@3ds.com
}

\author{
David Salinas \\ Gipsa-lab \\ Grenoble, France \\ david.salinas@gipsa- \\ lab.grenoble-inp.fr
}

\begin{abstract}
We associate with each compact set $X$ of $\mathbb{R}^{n}$ two real-valued functions $c_{X}$ and $h_{X}$ defined on $\mathbb{R}^{+}$which provide two measures of how much the set $X$ fails to be convex at a given scale. First, we show that, when $P$ is a finite point set, an upper bound on $c_{P}(t)$ entails that the Rips complex of $P$ at scale $r$ collapses to the Čech complex of $P$ at scale $r$ for some suitable values of the parameters $t$ and $r$. Second, we prove that, when $P$ samples a compact set $X$, an upper bound on $h_{X}$ over some interval guarantees a topologically correct reconstruction of the shape $X$ either with a Čech complex of $P$ or with a Rips complex of $P$. Regarding the reconstruction with Čech complexes, our work compares well with previous approaches when $X$ is a smooth set and surprisingly enough, even improves constants when $X$ has a positive $\mu$-reach. Most importantly, our work shows that Rips complexes can also be used to provide topologically correct reconstruction of shapes. This may be of some computational interest in high dimensions.
\end{abstract}

\section{Categories and Subject Descriptors}

F.2.2 [Analysis of Algorithms and Problem Complexity]: Nonnumerical Algorithms and Problems-Geometrical problems and computations, Computations on discrete structures; I.3.5 [Computer Graphics]: Computational Geometry and Object Modeling

\section{General Terms}

Theory, Algorithms

\section{Keywords}

Shape reconstruction, Vietoris-Rips complexes, clique complexes, Čech complexes, sampling, Euclidean spaces, metric spaces, homotopy equivalence, collapses

\footnotetext{
*This work is partially supported by ANR Project GIGA ANR-09BLAN-0331-01.
}

Permission to make digital or hard copies of all or part of this work for personal or classroom use is granted without fee provided that copies are not made or distributed for profit or commercial advantage and that copies bear this notice and the full citation on the first page. To copy otherwise, to republish, to post on servers or to redistribute to lists, requires prior specific permission and/or a fee.

SCG'11, June 13-15, 2011, Paris, France.

Copyright 2011 ACM 978-1-4503-0682-9/11/06 ...\$10.00.

\section{INTRODUCTION}

In this paper, we formulate conditions under which Rips complexes reconstruct shapes using measures of how far the shape is from being convex.

\section{Motivation.}

The problem of reconstructing shapes from point clouds arises in many fields, including computer graphics and machine learning 3 21]. Maybe one of the simplest reconstruction method is to output an $\alpha$-offset of the sample points, that is, the union of balls centered at the sample with radius $\alpha$. Assuming the shape is a smooth manifold [32 17] or more generally has a positive $\mu$-reach [15], it has been proved that this method provides indeed an approximation with the correct homotopy type for a sufficiently dense sample and a suitable value of the offset parameter $\alpha$. Topologically, this is equivalent to computing the $\alpha$-shape [23, 24] of the sample points, which can be obtained by first building the Delaunay triangulation and then keeping simplices that fit in an empty ball of radius $\alpha$ or less.

This approach works well for point clouds in three-dimensional space which have Delaunay triangulations of affordable size [5, 6]. But, as the dimension of the ambient space increases, the size of the Delaunay triangulation explodes [1] and other strategies must be found. If the data points lie on a low-dimensional submanifold, it seems reasonable to ask that the building of the reconstruction depends only upon the intrinsic dimension of the data. This motivated de Silva [19] to introduce witness complexes and Boissonnat and Ghosh [13] to define tangential Delaunay complexes. For medium dimensions, Boissonnat and al. [12] have modified the data structure representing the Delaunay complex and are able to manage complexes of reasonable size up to dimension six in practice. In particular, they avoid the explicit representation of all Delaunay simplices by storing only edges in what they call the Delaunay graph, an idea close to that of using Vietoris-Rips complexes developed in this paper.

\section{Vietoris-Rips complexes.}

Given a point set $P$ and a scale parameter $\alpha$, the Vietoris-Rips complex is the simplicial complex whose simplices are subsets of points in $P$ with diameter at most $2 \alpha$. Rips complexes are examples of flag complexes, and as such enjoy the property that a subset of $P$ belongs to the complex if and only if all its edges belong to the complex. In other words, Rips complexes are completely determined by the graph of their edges. This compressed form of storage makes Rips complexes very appealing for computations, at least in high dimensions. Recent results study their simplification through 
homotopy-preserving edge collapses [34, 35] and edge contractions [8]. However, the strategy of using Rips complexes makes sense only if they are able to reflect the topology of the shape that their vertices sample. A closely related family of simplicial complexes are Čech complexes. Specifically, the $\breve{C}$ ech complex of $P$ at scale $\alpha$ consists of all simplices spanned by points in $P$ that fit in a ball of radius $\alpha$. The Čech complex of $P$ at scale $\alpha$ is homotopy equivalent to the $\alpha$-offset of $P$ and therefore also possesses the ability to reproduce the topology of the shape sampled by $P$. This property was used by Chazal and Oudot [18] to extract topological information on the shape from the Rips complex filtration, by interleaving it with the Čech complex filtration and using persistence topology.

The main contribution of this paper is to unveil a more direct relationship between the respective topologies of the Rips complex and the sampled shape. Specifically, we give conditions under which Rips complexes capture the topology of the shape. In a different setting, it has been proved in [28 29] that the Rips complex of a point set close enough to a Riemannian manifold for the Gromov-Hausdorff distance shares the homotopy type of the manifold. However, these results focus on smooth manifolds, consider the intrinsic Riemannian metric instead of the Euclidean ambient metric and are not effective since they do not give explicit constants. Nevertheless, they suggest that Rips complexes could be used in practice to produce topologically correct approximations of shapes.

Partially related to our work, we should mention [14] which relates the fundamental group of a Rips complex and its shadow (see below) in dimension 2 and give counterexamples in higher dimensions.

\section{Sampling conditions.}

In any case, it is necessary for a point cloud to be accurate and dense enough to reflect the topology of the shape it samples. The quality of the sample is typically expressed in terms of Hausdorff distance to the shape. Guaranteed reconstruction methods are generally accompanied by results of the following form: if the Hausdorff distance is smaller than some notion of topological feature size of the shape, then the output is topologically correct. First sampling conditions were expressed in terms of the reach, which is the infimum of distances between points in the shape and points in its medial axis [4, 2, 11, 32, 17|. Unfortunately, the reach vanishes on sharp concave edges and therefore is not suitable for expressing sampling conditions for non-smooth manifolds or stratified objects. To deal with this problem, authors in [15] introduce a new characterization of the feature size, the $\mu$-reach, which allows them to formulate sampling conditions for a large class of non-smooth compact subsets of Euclidean space.

In this work, we introduce two new measures of feature size, both called convexity defects. Roughly speaking, they measure how far an object is from being locally convex, in the same manner as curvature measures how far an object is from being locally flat. In Section 4, we use these measures to express sampling conditions first for the Čech complex and second for the Rips complex. Regarding the reconstruction with Čech complexes, our work compares well with previous approaches when $X$ is a smooth set and surprisingly enough, even improve constants when $X$ has a positive $\mu$-reach. Most importantly, this new framework allows us to prove that Rips complexes also provide topologically correct reconstruction, assuming shapes have a positive $\mu$-reach, for $\mu$ sufficiently large. For this, we first find conditions under which Rips complexes collapse to Čech complexes in Section 3

\section{PRELIMINARIES}

In this section, we introduce the definitions and tools we need to state and prove our results.

\subsection{Metric space and distances}

Throughout this paper, we shall consider subsets of the Euclidean $n$-space $\mathbb{R}^{n}$ for $n \geq 1$. The Euclidean distance between two points $x$ and $y$ of $\mathbb{R}^{n}$ is denoted $\|x-y\|$. Given two subsets $X$ and $Y$ of $\mathbb{R}^{n}$, we write $d_{H}(Y \mid X)=\sup _{y \in Y} d(y, X)$ for the one-sided Hausdorff distance of $Y$ from $X$, where $d(y, X)$ is the infimum of the Euclidean distances between $y$ and points $x$ in $X$. Observe that $d_{H}(Y \mid X) \leq \varepsilon$ if and only if $Y$ is contained in the $\varepsilon$-offset $X^{\varepsilon}=\left\{y \in \mathbb{R}^{n} \mid d(y, X) \leq \varepsilon\right\}$. The Hausdorff distance between $X$ and $Y$ is $d_{H}(X, Y)=\max \left\{d_{H}(X \mid Y), d_{H}(Y \mid X)\right\}$. The closed ball with center $z$ and radius $r$ is denoted $B(z, r)$. Balls will always be assumed to be closed, unless stated otherwise.

\subsection{Smallest enclosing ball}

Recall that the diameter of a subset $\sigma$ of $\mathbb{R}^{n}$ is the supremum of distances between pairs of points in $\sigma$, which we denote as $\operatorname{Diam}(\sigma)=\sup _{p, q \in \sigma}\|p-q\|$. A subset $\sigma$ is said to be bounded if its diameter is finite. It is well known that the smallest ball enclosing a non-empty bounded set $\sigma$ of $\mathbb{R}^{n}$ is well-defined (see the appendix for a proof). We denote its center by $\operatorname{Center}(\sigma)$ and its radius by $\operatorname{Rad}(\sigma)$. Writing $\operatorname{Hull}(X)$ for the convex hull of $X$ and $\bar{X}$ for the closure of $X$, it is not hard to check (by contradiction) that $\operatorname{Center}(\sigma) \in \overline{\operatorname{Hull}(\sigma)}$. We now give a key property of the smallest enclosing ball. Stabilities of its radius and center are established in the appendix.

LEMMA 1. For any non-empty bounded subset $\sigma \subset \mathbb{R}^{n}$, any point $x \in \mathbb{R}^{n}$ and any point $y \in \operatorname{Hull}(\sigma)$, we have that $d(x, \sigma)^{2} \leq$ $\|x-y\|^{2}+\operatorname{Rad}(\sigma)^{2}-\|y-\operatorname{Center}(\sigma)\|^{2}$.

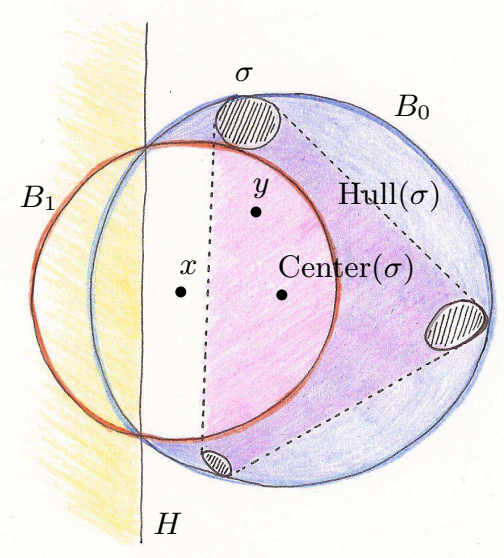

Figure 1: Notations for the proof of Lemma 1.

PROOF. Suppose $d(x, \sigma)>\|x-y\|$ for otherwise the result is clear. Let $B_{0}$ be the smallest ball enclosing $\sigma$ and let $B_{1}$ be the largest ball centered at $x$ whose interior does not intersect $\sigma$; see Figure 1. By construction, $\sigma \subset B_{0} \backslash B_{1}$. Recall that the power distance of a point $y$ from a ball $B$ is $\pi_{B}(y)=\|y-z\|^{2}-r^{2}$, where $z$ is the center of $B$ and $r$ its radius. Let $H$ be the set of points whose power distance to $B_{0}$ is at most as large as the power distance to $B_{1} . H$ is a closed half-space which contains the set difference $B_{0} \backslash B_{1}$. In particular, it contains $\sigma$ and any point $y \in$ $\operatorname{Hull}(\sigma)$. Thus, $\pi_{B_{0}}(y) \leq \pi_{B_{1}}(y)$ and the result follows. 


\subsection{Abstract simplicial complexes}

Let $P$ be a finite set of points in $\mathbb{R}^{n}$. We call any non-empty subset $\sigma \subset P$ an abstract simplex. Its dimension is one less than its cardinality. A $i$-simplex is an abstract simplex of dimension $i$. If $\tau \subset \sigma$ is a non-empty subset, we call $\tau$ a face of $\sigma$ and $\sigma$ a coface of $\tau$. An abstract simplicial complex $K$ is a collection of non-empty abstract simplices that contains, with every simplex, the faces of that simplex. The vertex set of the abstract simplicial complex $K$ is the union of its elements, $\operatorname{Vert}(K)=\bigcup_{\sigma \in K} \sigma$. A subcomplex of $K$ is a simplicial complex $L \subset K$. A particular subcomplex is the $i$-skeleton consisting of all simplices of dimension $i$ or less, which we denote by $K^{(i)}$. The shadow of $K$ is the subset of $\mathbb{R}^{n}$ covered by the convex hull of simplices in $K$, Shd $K=\bigcup_{\sigma \in K} \operatorname{Hull}(\sigma)$, not to be confused with $|K|$, the underlying space of a geometric realization of $K$; see [31]. If $N$ is the cardinal of the vertex set $\operatorname{Vert}(K)$ of $K$ and if $f: \operatorname{Vert}(K) \rightarrow \mathbb{R}^{N}$ sends $\operatorname{Vert}(K)$ to an affinely independant set $f(\operatorname{Vert}(K))$, then $|K|=\bigcup_{\sigma \in K} \operatorname{Hull}(f(\sigma))$ (up to a homeomorphism). Generally, $|K|$ and Shd $K$ are not homeomorphic since the relative interiors of the convex hulls of two different simplices of $K$ may overlap.

We now review two natural ways of constructing an abstract simplicial complex, given as input a finite set of points in $\mathbb{R}^{n}$ and a feature scale parameter $t \geq 0$. The definitions given below may change from one author to another.

The Čech complex $\mathcal{C}(P, t)$ is the abstract simplicial complex whose $k$-simplices correspond to subsets of $k+1$ points that can be enclosed in a ball of radius $t, \mathcal{C}(P, t)=\{\sigma \mid \emptyset \neq \sigma \subset$ $P, \operatorname{Rad}(\sigma) \leq t\}$. Equivalently, a $k$-simplex $\left\{p_{0}, \ldots, p_{k}\right\}$ belongs to the Čech complex if and only if the $k+1$ closed Euclidean balls $B\left(p_{i}, t\right)$ have non-empty common intersection. Let Nrv $F=$ $\{G \subset F \mid \bigcap G \neq \emptyset\}$ denote the nerve of the collection $F$. The Cech complex is the nerve of the collection of balls $\{B(p, t) \mid p \in$ $P\}$. Since balls are convex, the Nerve Lemma [10 25] implies that the Čech complex $\mathcal{C}(P, t)$ is homotopy equivalent to the union of these balls, that is, $|\mathcal{C}(P, t)| \simeq P^{t}$.

The Vietoris-Rips complex is a variant of the Čech complex which is easier to compute. The Vietoris-Rips complex, $\mathcal{R}(P, t)$ is the abstract simplicial complex whose $k$-simplices correspond to subsets of $k+1$ points in $P$ with diameter at most $2 t, \mathcal{R}(P, t)=\{\sigma \mid \emptyset \neq$ $\sigma \subset P, \operatorname{Diam}(\sigma) \leq 2 t\}$. For simplicity, we refer to $\mathcal{R}(P, t)$ as the Rips complex. Recall that the flag complex of a graph $G$, denoted Flag $G$, is the maximal simplicial complex whose 1-skeleton is $G$. The Rips complex is an example of a flag complex. More precisely, this is the largest simplicial complex sharing with the Cech complex the same 1-skeleton, $\mathcal{R}(P, t)=\operatorname{Flag}\left(\mathcal{C}(P, t)^{(1)}\right)$. Generally, $\mathcal{R}(P, t)$ and $\mathcal{C}(P, t)$ do not share the same topology. It follows that the Rips complex $\mathcal{R}(P, t)$ is generally not homotopy equivalent to the $t$-offset $P^{t}$. Our goal in the next section is to find a condition on the point set $P$ which guarantees that $|\mathcal{R}(P, t)| \simeq|\mathcal{C}(P, t)|$ and $|\mathcal{R}(P, t)| \simeq P^{t}$. Along the way, we will need a result in $|20|$ which is a consequence of Jung's Theorem and which says that there is chain of inclusion

$$
\mathcal{C}(P, t) \subset \mathcal{R}(P, t) \subset \mathcal{C}\left(P, \vartheta_{n} t\right) \quad \text { where } \vartheta_{n}=\sqrt{\frac{2 n}{n+1}} .
$$

\section{FROM RIPS TO ČECH COMPLEXES}

In this section, we introduce two functions that one can associate with any non-empty bounded subset $X \subset \mathbb{R}^{n}$ and that provide two different ways of measuring convexity defects of $X$. Based on these functions, we will be able in Section 3.3 to formulate a condition which suffices to guarantee that Rips complexes of a finite set of points $P$ deformation retract to Čech complexes of $P$ using a new kind of collapses described in Section 3.2

\subsection{Convexity defects measures}

To avoid lengthy sentences, we adopt the convention that $X$ is always assumed to be non-empty and bounded in this section. In particular, any non-empty subset $\sigma \subset X$ is also bounded and thus has a well-defined smallest enclosing ball. Recalling that $\operatorname{Hull}(X)$ denotes the convex hull of $X$, we first extend the notion of convex hull. We define the convex hull of $X$ at scale $t$ as the subset (see Figure 2,

$$
\operatorname{Hull}(X, t)=\bigcup_{\substack{\emptyset \neq \sigma \subset X \\ \operatorname{Rad}(\sigma) \leq t}} \operatorname{Hull}(\sigma) .
$$

Note that if $P$ is a finite set of points, then $\operatorname{Hull}(P, t)$ is the shadow of the Čech complex $\mathcal{C}(P, t)$. Similarly, we define the set of centers of $X$ at scale $t$ as the subset:

$$
\operatorname{Centers}(X, t)=\bigcup_{\substack{\sigma \neq \sigma \subset X \\ \operatorname{Rad}(\sigma) \leq t}}\{\operatorname{Center}(\sigma)\}
$$

DEFINITION 1 (CONVEXITY DEFECTS FUnCTIONS). Given a subset $X \subset \mathbb{R}^{n}$, we associate to $X$ two real-valued functions $h_{X}: \mathbb{R}_{+} \rightarrow \mathbb{R}_{+}$defined by $h_{X}(t)=d_{H}(\operatorname{Hull}(X, t) \mid X)$ and $c_{X}: \mathbb{R}_{+} \rightarrow \mathbb{R}_{+}$defined by $c_{X}(t)=d_{H}(\operatorname{Centers}(X, t) \mid X)$.

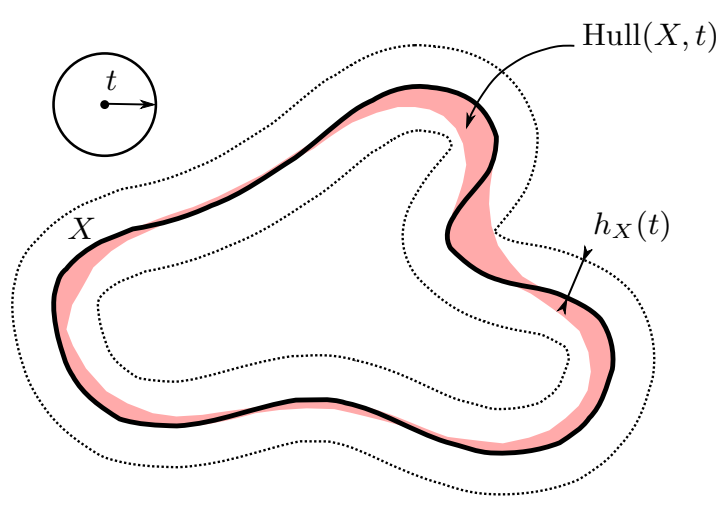

Figure 2: Smallest offset of $X$ containing $\operatorname{Hull}(X, t)$.

Intuitively, $h_{X}$ and $c_{X}$ can be thought of as functions that measure the convexity defects of $X$ at a given scale. To make this idea precise, observe that if $X \subset \mathbb{R}^{n}$ is compact, then $X$ is convex if and only if $h_{X}=0$. Similarly, a compact set $X$ is convex if and only if $c_{X}=0$. The two convexity functions $h_{X}$ and $c_{X}$ will play a different role. While $c_{P}$ is all we need to study the Rips complex of a finite point set $P$ in Section 3.3, it turns out that $h_{X}$ is more stable than $c_{X}$ and will be used in Section 4.2 to express sampling conditions in reconstruction theorems.

Let us make a few remarks. Because $X$ is a subset of both $\operatorname{Hull}(X, t)$ and Centers $(X, t)$, the two one-sided Hausdorff distances $d_{H}(X \mid \operatorname{Hull}(X, t))$ and $d_{H}(X \mid \operatorname{Centers}(X, t))$ vanish. It follows that we could have used in the above definition two-sided Hausdorff distances instead of one-sided Hausdorff distances. We now list a few basic properties of functions $h_{X}$ and $c_{X}$ (see Figure 5. First, $h_{X}$ and $c_{X}$ both vanish at 0 , are increasing in the interval $[0, \operatorname{Rad}(X)]$ and become constant above $\operatorname{Rad}(X)$. Since $\operatorname{Center}(\sigma) \in \overline{\operatorname{Hull}(\sigma)}$, we have $c_{X} \leq h_{X}$. It is easy to check that for a subset $X \subset \mathbb{R}^{n}$ and two non-negative real numbers $t$ and $\alpha$, 
the following three conditions are equivalent: (1) $h_{X}(t) \leq \alpha$; (2) $\operatorname{Hull}(X, t) \subset X^{\alpha} ;(3)\left[\operatorname{Rad}(\sigma) \leq t \Longrightarrow \operatorname{Hull}(\sigma) \subset X^{\alpha}\right]$ for all $\sigma \subset X$. In particular, we get that $h_{X}(t) \leq t$ for all $t \geq 0$ since $\operatorname{Rad}(\sigma) \leq t \Longrightarrow \operatorname{Hull}(\sigma) \subset \sigma^{t}$ by Lemma 1 applied for $x=y$.

\subsection{Collapses}

This section describes collapses that will be useful to deformation retract Rips complexes to Cech complexes in the next section.

First, we need some definitions. Let $\sigma$ be a simplex of the simplicial complex $K$. The star of $\sigma$ in $K$, denoted $\operatorname{St}_{K}(\sigma)$, is the collection of simplices of $K$ having $\sigma$ as a face. The closure of $\mathrm{St}_{K}(\sigma)$ is denoted $\overline{\mathrm{St}}_{K}(\sigma)$; it is the smallest simplicial complex containing $\operatorname{St}_{K}(\sigma)$. The link of $\sigma$ in $K$, denoted $\operatorname{Lk}_{K}(\sigma)$, is the collection of simplices of $K$ lying in $\overline{\operatorname{St}}_{K}(\sigma)$ that are disjoint from $\sigma$. Given two non-empty simplicial complexes $K$ and $L$, the smallest simplicial complex containing all the simplices of the form $\kappa \cup \lambda$ where $\kappa \in K$ and $\lambda \in L$ is called the join of $K$ and $L$ and is denoted by $K * L$. We adopt the convention that if $L$ is empty, then $K * L=K$. A simplicial complex $K$ is said to be a cone if it contains a vertex $o$ such that the following implication holds: $\sigma \in K \Longrightarrow \sigma \cup\{o\} \in K$. Equivalently, a cone is the join $o * L$ of a (possibly empty) simplicial complex $L$ and a vertex $o \notin L$. The vertex $o$ is called the apex of the cone. By definition a cone can never be empty since it always contains at least its apex.
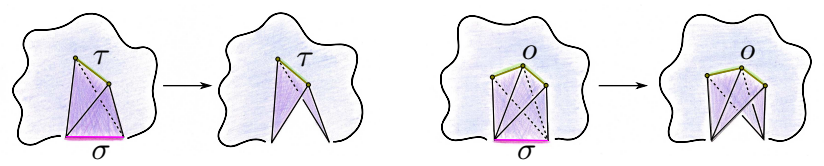

Figure 3: Left: In a classical collapse, the link of $\sigma$ has a unique inclusion-maximal simplex $\tau$. Right: In an extended collapse, the link of $\sigma$ is a cone with apex $o$.

Given a simplicial complex $K$, we are interested in the operation that removes the entire star of a simplex $\sigma \in K$ (see Figure 3 . Provided that there is a unique inclusion-maximal simplex $\tau \neq \sigma$ in the star of $\sigma$, it is well-known that $|K|$ deformation retracts to $\left|K \backslash \operatorname{St}_{K}(\sigma)\right|$ and the operation that removes $\operatorname{St}_{K}(\sigma)$ is then called a collapse [22]. Following and extending what was done in [9], we still call a collapse the operation that removes $\operatorname{St}_{K}(\sigma)$ assuming the weaker condition that the link of $\sigma$ is a cone. Our terminology finds its justification in the following lemma.

LEMMA 2. Let $K$ be a simplicial complex and let $\sigma$ be a simplex of $K$. If the link of $\sigma$ is a cone, then $|K|$ deformation retracts to $\left|K \backslash \mathrm{St}_{K}(\sigma)\right|$.

Proof. Suppose first that $K$ contains a vertex $v$ whose link is a cone with apex $o$. Slightly adapting the proof of Proposition 2.9 in [9], we prove that $|K|$ deformation retracts to $\left|K \backslash \mathrm{St}_{K}(v)\right|$. Define a vertex map $\pi: \operatorname{Vert}(K) \rightarrow \operatorname{Vert}(K)$ which is the identity on $\operatorname{Vert}(K) \backslash\{v\}$ and such that $\pi(v)=o$. If $\tau$ is a proper coface of $v$, then $\tau \backslash\{v\}$ belongs to the link of $v$ and because the link is a cone with apex $o$, it also contains $\pi(\tau)=(\tau \backslash\{v\}) \cup\{o\}$. Moreover, $\pi(\tau) \cup \tau=\tau \cup\{o\}$ belongs to $K$. It follows that $\pi$ can be extended to a simplicial map which is contiguous (see [31] for a definition) to the identity of $K$. Furthermore, $\pi(K)=K \backslash \mathrm{St}_{K}(v)$ and the restriction of $\pi$ to $K \backslash \mathrm{St}_{K}(v)$ is the identity. Thus, the map $H:|K| \times[0,1] \rightarrow|K|$ defined by $H(x, t)=(1-t) x+t \pi(x)$ is a deformation retraction of $|K|$ onto $\left|K \backslash \operatorname{St}_{K}(v)\right|$.

Suppose now $\sigma$ is a simplex in $K$ whose link is a cone with apex $o$. We reduce this case to the previous one by subdividing simplices in the star of $\sigma$ as follows. Let $\hat{\sigma}$ be the barycenter of $\sigma$ and let $\operatorname{Bd} \sigma$ designate the set of proper faces of $\sigma$. We build a simplicial complex $K^{\prime}$ from $K$, replacing the closed star $\overline{\operatorname{St}}_{K}(\sigma)$ by the join $\{\hat{\sigma}\} * \operatorname{Bd} \sigma * \operatorname{Lk}_{K}(\sigma)$. Note that if $\sigma$ is a vertex, then the join coincides with the closed star of $\sigma$ and $K^{\prime}=K$. By construction, the simplicial complex $K$ and its subdivision $K^{\prime}$ have in common the set of simplices $K \backslash \mathrm{St}_{K}(\sigma)=K^{\prime} \backslash \mathrm{St}_{K^{\prime}}(\hat{\sigma})$. Let us show that the link of $\hat{\sigma}$ in $K^{\prime}$ is a cone with apex $o$. By construction, $\operatorname{Lk}_{K^{\prime}}(\hat{\sigma})=\operatorname{Bd} \sigma * \operatorname{Lk}_{K}(\sigma)$. Using the existence of a subcomplex $L \subset K$ such that $\operatorname{Lk}_{K}(\sigma)=\{o\} * L$, we get that $\operatorname{Lk}_{K^{\prime}}(\hat{\sigma})=\{o\} * \mathrm{Bd} \sigma * L$ is a cone. The first part of the proof implies that $|K|=\left|K^{\prime}\right|$ deformation retracts to $\left|K \backslash \mathrm{St}_{K}(\sigma)\right|=$ $\left|K^{\prime} \backslash \operatorname{St}_{K^{\prime}}(\hat{\sigma})\right|$.

\subsection{Almost Rips complexes}

In this section, we introduce a 2-parameter family of Rips complexes and give the precise condition on a finite point set for which we can prove that a Rips complex in this family deformation retracts to a Čech complex. As a consequence, we also state conditions under which a Čech complex deformation retracts to another one. Let us first define a 2-parameter family that contains prior Rips complexes as a subfamily:

DEFINITION 2. For any point set $P \subset \mathbb{R}^{n}$ and any real numbers $\alpha, \beta \geq 0$ with $\alpha \leq \beta$, we call the flag complex of any graph $G$ satisfying $\mathcal{R}(P, \alpha) \subset$ Flag $G \subset \mathcal{R}(P, \beta)$ an $(\alpha, \beta)$-almost Rips complex of $P$.

In other words, the simplicial complex Flag $G$ is an $(\alpha, \beta)$-almost Rips complex of $P$ if and only if every pairs of points in $P$ within distance $2 \alpha$ are connected by an edge in $G$ and no edge of $G$ has length larger than $2 \beta$. Equivalently, for every pairs $(p, q) \in P^{2}$, $\|p-q\| \leq 2 \alpha$ implies $p q \in G$ and $\|p-q\|>2 \beta$ implies $p q \notin G$. In particular, $K$ is an $(\alpha, \alpha)$-almost Rips complex of $P$ if and only if $K=\mathcal{R}(P, \alpha)$. To state our main theorem, it is convenient to define $\alpha$ to be an inert value of $P$ if $\operatorname{Rad}(\sigma) \neq \alpha$ for all non-empty subsets $\sigma \subset P$. The finiteness of $P$ implies that $P$ has only finitely many non-inert values. Thus, assuming $\alpha$ to be inert is not a too restrictive hypothesis.

THEOREM 1. Let $P \subset \mathbb{R}^{n}$ be a finite set of points. For any real numbers $\beta \geq \alpha \geq 0$ such that $\alpha$ is an inert value of $P$ and $c_{P}\left(\vartheta_{\mathrm{n}} \beta\right)<2 \alpha-\vartheta_{\mathrm{n}} \beta$, there exists a sequence of collapses from any $(\alpha, \beta)$-almost Rips complex of $P$ to the Čech complex $\mathcal{C}(P, \alpha)$.

Proof. Let $G$ be a graph whose flag complex is an $(\alpha, \beta)$ almost Rips complex of $P$. For $t \geq 0$, consider the simplicial complex $\mathcal{F}(t)=\mathcal{C}(P, t) \cap$ Flag $G$. Clearly, we have the chain of inclusions:

$$
\mathcal{C}(P, \alpha) \subset \mathcal{R}(P, \alpha) \subset \operatorname{Flag} G \subset \mathcal{R}(P, \beta) \subset \mathcal{C}\left(P, \vartheta_{\mathrm{n}} \beta\right)
$$

and therefore $\mathcal{F}(\alpha)=\mathcal{C}(P, \alpha)$ and $\mathcal{F}\left(\vartheta_{\mathrm{n}} \beta\right)=\operatorname{Flag} G$. As we continuously increase the feature parameter $t$ from $\alpha$ to $\vartheta_{\mathrm{n}} \beta$, we get a finite family of nested Čech complexes:

$$
\mathcal{C}(P, \alpha)=\mathcal{C}_{0} \subset \mathcal{C}_{1} \subset \cdots \subset \mathcal{C}_{k}=\mathcal{C}\left(P, \vartheta_{\mathrm{n}} \beta\right) .
$$

For $0<i<k$, let $t_{i}$ be the smallest value of $t$ such that $\mathcal{C}_{i}=$ $\mathcal{C}(P, t)$ and set $\mathcal{F}_{i}=\mathcal{F}\left(t_{i}\right)$. Correspondingly, we get a 1-parameter family of simplicial complexes:

$$
\mathcal{C}(P, \alpha)=\mathcal{F}_{0} \subset \mathcal{F}_{1} \subset \cdots \subset \mathcal{F}_{k}=\operatorname{Flag} G .
$$

Let us first assume that $P$ satisfies the two generic conditions $(\star)$ and $(\star \star)$ instead of the condition that $\operatorname{Rad}(\sigma) \neq \alpha$ for all nonempty subsets $\sigma \subset P$ : 
(*) For all simplices $\sigma, \tau \subset P$, if $\operatorname{Rad}(\sigma)=\operatorname{Rad}(\tau)$ then $\operatorname{Center}(\sigma)=\operatorname{Center}(\tau)$;

$(\star \star)$ For any ball $B$, the set of simplices in $P$ that have $B$ as a smallest enclosing ball is either empty or has a unique inclusion-minimal element.

Under these two conditions, we prove the theorem by first showing that $\mathcal{C}_{i}$ collapses to $\mathcal{C}_{i-1}$ for all $0<i \leq k$ and secondly that $\mathcal{F}_{i}$ is either equal or collapses to $\mathcal{F}_{i-1}$ for all $0<i \leq k$. Because of condition $(\star)$, all simplices in the set difference $\mathcal{C}_{i} \backslash \mathcal{C}_{i-1}$ share the same smallest enclosing ball $B\left(z_{i}, t_{i}\right)$. Because of condition $(\star \star)$, the set of simplices sharing the same smallest enclosing ball $B\left(z_{i}, t_{i}\right)$ has a unique inclusion-minimal element $\sigma_{i}$. Suppose $\eta$ is a coface of $\sigma_{i}$ in $\mathcal{C}_{i}$. Since $\sigma_{i} \subset \eta$, we deduce that $t_{i}=\operatorname{Rad}\left(\sigma_{i}\right) \leq$ $\operatorname{Rad}(\eta)$ and therefore $\eta \in \mathcal{C}_{i} \backslash \mathcal{C}_{i-1}$. Hence, $\mathcal{C}_{i} \backslash \mathcal{C}_{i-1}$ is the star of $\sigma_{i}$ in $\mathcal{C}_{i}$. Note that the simplex $\tau_{i}=\left\{p \in P \mid\left\|z_{i}-p\right\| \leq\right.$ $\left.t_{i}\right\}$ obtained by gathering all points of $P$ in $B\left(z_{i}, t_{i}\right)$ belongs to $\mathcal{C}_{i} \backslash \mathcal{C}_{i-1}$ and is the unique inclusion-maximal simplex in this set difference (see Figure 4, left). To prove that $\mathcal{C}_{i}$ collapses to $\mathcal{C}_{i-1}$, it remains to establish that $\sigma_{i} \neq \tau_{i}$. By choice of $\sigma_{i}$ as an inclusionminimal element amongst simplices with smallest enclosing ball $B\left(z_{i}, t_{i}\right)$, the vertices of $\sigma_{i}$ all lie on the sphere with center $z_{i}$ and radius $t_{i}$. On the other hand, by definition of $c_{P}\left(t_{i}\right)$ as the onesided Hausdorff distance of the centers of $P$ at scale $t_{i}$ from $P$, there exists at least a point $o$ of $P$ at distance $c_{P}\left(t_{i}\right)$ or less from the center $z_{i}$. Since $c_{P}\left(t_{i}\right) \leq c_{P}\left(\vartheta_{\mathrm{n}} \beta\right)<\alpha \leq t_{i}$, the point $o$ belongs to the interior of $B\left(z_{i}, t_{i}\right)$. Thus, $o \notin \sigma_{i}, o \in \tau_{i}$, and therefore $\sigma_{i} \neq \tau_{i}$, showing that $\mathcal{C}_{i}$ collapses to $\mathcal{C}_{i-1}$.
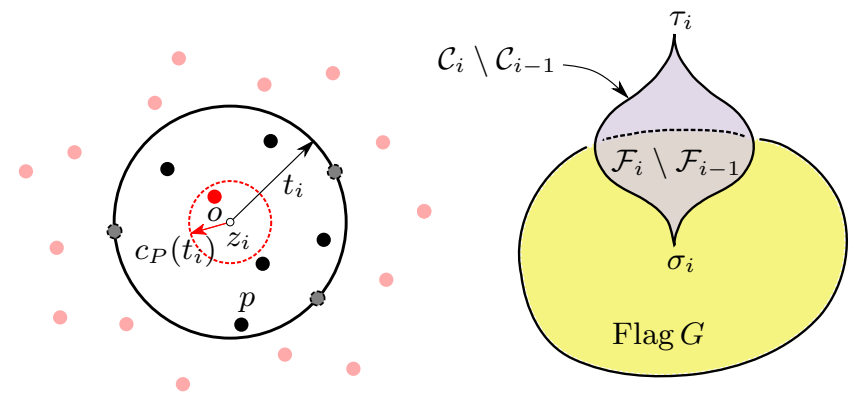

Figure 4: Notations for the proof of Theorem 1. Left: $\tau_{i}$ is the simplex whose vertices are points of $P$ in $B\left(z_{i}, t_{i}\right) . \sigma_{i}$ is the face obtained by keeping vertices on the boundary of $B\left(z_{i}, t_{i}\right)$. Right: Schematic representation of simplices in $\mathcal{C}_{i} \backslash \mathcal{C}_{i-1}$.

Let us now turn our attention to $\mathcal{F}_{i}$ and $\mathcal{F}_{i-1}$. If $\sigma_{i} \notin \mathcal{F}_{i}$, then $\mathcal{F}_{i}=\mathcal{F}_{i-1}$. If $\sigma_{i} \in \mathcal{F}_{i}$, the star of $\sigma_{i}$ in $\mathcal{F}_{i}$ is equal to the star of $\sigma_{i}$ in $\mathcal{C}_{i}$ intersection the flag of $G$ and $\mathcal{F}_{i-1}=\mathcal{F}_{i} \backslash \operatorname{St}_{\mathcal{F}_{i}}\left(\sigma_{i}\right)$ (see Figure 4 right). Let us prove that the link of $\sigma_{i}$ in $\mathcal{F}_{i}$ is a cone with apex $o$, which guarantees that $\mathcal{F}_{i}$ collapses to $\mathcal{F}_{i-1}$. Suppose $\eta$ is a coface of $\sigma_{i}$ in $\mathcal{F}_{i}$ and let us show that $\eta \cup\{o\}$ is also a coface. Clearly, $\eta \cup\{o\}$ belongs to the Čech complex $\mathcal{C}_{i}$ since for all points $p \in \eta \cup\{o\},\left\|z_{i}-p\right\| \leq t_{i}$. Let us prove that $\eta \cup\{o\}$ also belongs to Flag $G$. Since $\eta$ belongs to Flag $G$, it suffices to prove that all edges connecting $o$ to a vertex $p$ of $\eta$ have length $2 \alpha$ or less. Indeed, for all points $p \in \eta$, we have

$$
\|p-o\| \leq\left\|z_{i}-p\right\|+\left\|z_{i}-o\right\| \leq t_{i}+c_{P}\left(t_{i}\right) \leq 2 \alpha
$$

showing that $\eta \cup\{o\} \in$ Flag $G$. Hence, $\eta \cup\{o\}$ belongs to $\mathcal{F}_{i}$. Setting $\eta=\sigma_{i}$, we get that $\sigma_{i} \cup\{o\}$ is a coface of $\sigma_{i}$ and since $o \notin \sigma_{i}$, it follows that $\{o\}$ belongs to the link of $\sigma_{i}$ in $\mathcal{F}_{i}$. Hence, the link of $\sigma_{i}$ in $\mathcal{F}_{i}$ is a cone, which concludes the proof of Theorem
1 assuming generic conditions $(\star)$ and $(\star \star)$ instead of the condition $\operatorname{Rad}(\sigma) \neq \alpha$ for all non-empty subsets $\sigma \subset P$.

If $P$ does not satisfy the generic conditions $(\star)$ and $(\star \star)$, we use Lemma 10 to find a perturbation $f$ of the points such that $f(P)$ satisfies $(\star)$ and $(\star \star)$ and conditions $(i),($ ii $)$ and $($ iii $)$ of Lemma 10 for some $\beta^{\prime}>\beta$. Applying Theorem 1 to $f(P)$ with the values $\alpha$ and $\beta^{\prime}$, we get that there exists a sequence of collapses from the $\left(\alpha, \beta^{\prime}\right)$-almost Rips complex Flag $f(G)=f(\operatorname{Flag}(G))$ to the Cech complex $\mathcal{C}(f(P), \alpha)=f(\mathcal{C}(P, \alpha))$. Hence, the theorem also holds in the non-generic case.

Choosing $\beta=\alpha$ in the theorem gives conditions under which $|\mathcal{R}(P, \alpha)| \simeq|\mathcal{C}(P, \alpha)| \simeq P^{\alpha}$. Figure 6 provides a graphical representation of the hypothesis of the theorem. Slightly adapting the first part of the proof we get the following result:

THEOREM 2. Let $\beta \geq \alpha \geq 0$ and let $P$ be a finite set of points of $\mathbb{R}^{n}$. If $\alpha$ is an inert value of $P$ and $c_{P}(t)<t$ for all $t \in[\alpha, \beta]$, then there exists a sequence of collapses from $\mathcal{C}(P, \beta)$ to $\mathcal{C}(P, \alpha)$.

\section{SHAPE RECONSTRUCTION}

In this section, we are interested in reconstructing a compact set $X \subset \mathbb{R}^{n}$ only known through a finite set of possibly noisy points $P \subset \mathbb{R}^{n}$. Using the convexity defect function $h_{X}$, we formulate two sampling conditions which guarantee respectively that the Čech complex and the Rips complex of $P$ are homotopy equivalent to any arbitrarily small offset of $X$ (Section 4.2). This requires us to study in more details convexity defects functions, establishing connections with the distance function to $X$ in Section 4.1 and the stability of $h_{X}$. Finally, we construct a bridge between shapes with an upper bounded convexity defects function and shapes with a positive $\mu$-reach in Section 4.3 We then compute in Section 4.4 the lowest density of points authorized by our theorems for a correct reconstruction.

\subsection{Characterizing critical values of the dis- tance function}

We begin by giving two characterizations of the critical values of the distance function to a compact set $X \subset \mathbb{R}^{n}$, based respectively on the two convexity defects functions $c_{X}$ and $h_{X}$. For this, we need some definitions. The distance function $d(\cdot, X)$ to the compact set $X \subset \mathbb{R}^{n}$ maps every point $y \in \mathbb{R}^{n}$ to its Euclidean distance to $X, d(y, X)=\min _{x \in X}\|x-y\|$. Although the distance function is not differentiable, it is possible to define a notion of critical points analogue to the classical one for differentiable functions. Specifically, Grove defines in [27, page 360] critical points for the distance function to a closed subset of a Riemannian manifold. Using Equation (1.1)' in 27. page 360], we recast this definition in our context as follows. Let $\Gamma_{X}(y)=\{x \in X \mid d(y, X)=\|x-y\|\}$ be the set of points in $X$ closest to $y$ :

Definition 3. We say that $y \in \mathbb{R}^{n}$ is a critical point of the distance function $d(\cdot, X)$ if $y \in \operatorname{Hull}\left(\Gamma_{X}(y)\right)$. The critical values of $d(\cdot, X)$ are the images by $d(\cdot, X)$ of its critical points.

Slightly recasting Proposition 1.8 in [27 page 362], we have:

Theorem 3 (Isotopy THEOREM [27]). Let $X \subset \mathbb{R}^{n}$ be a compact set and let $\beta \geq \alpha>0$ be two real numbers. If the distance function $d(\cdot, X)$ has no critical value in the interval $[\alpha, \beta]$, then $X^{\beta}$ deformation retracts to $X^{\alpha}$.

In section 3.1. we noted that $c_{X}(t) \leq h_{X}(t) \leq t$ for all $t$. Next lemma establishes that equality is attained if and only if $t$ is a critical value of the distance function to $X$ (see Figure 5). 
LEMMA 3. For any compact set $X \subset \mathbb{R}^{n}$ and any real number $t>0$, the following three conditions are equivalent: (1) $t$ is a critical value of $d(\cdot, X) ;(2) c_{X}(t)=t$; (3) $h_{X}(t)=t$.

Proof. Making $x=y$ in Lemma 1, we observe that if $y \in$ $\operatorname{Hull}(\sigma)$ satisfies $d(y, \sigma) \geq t$ and $\operatorname{Rad}(\sigma) \leq t$, then $y=\operatorname{Center}(\sigma)$.

Let us prove that (1) $\Longrightarrow(2)$. Consider a critical point $y$ whose distance to $X$ is $t$. Setting $\sigma=\Gamma_{X}(y)$, we have $y \in \operatorname{Hull}(\sigma)$, $d(y, \sigma)=t$ and $\operatorname{Rad}(\sigma) \leq t$. Thanks to our observation, it follows that $y=\operatorname{Center}(\sigma)$ and consequently $c_{X}(t)=t$. Because $c_{X}(t) \leq h_{X}(t) \leq t$, we have (2) $\Longrightarrow$ (3). Let us prove that (3) $\Longrightarrow$ (1). In other words, suppose $h_{X}(t)=t$ and let us prove that $t$ is a critical value of $d(\cdot, X)$. Since $X$ is compact, $h_{X}(t)=t$ means that we can find a compact set $\emptyset \neq \sigma \subset X$ with $\operatorname{Rad}(\sigma) \leq t$ and $y \in \operatorname{Hull}(\sigma)$ such that $t=d(y, X) \leq d(y, \sigma)$. Our observation then implies that $y=\operatorname{Center}(\sigma), t=\operatorname{Rad}(\sigma)$ and $\sigma$ represents a set of points in $X$ with minimum distance to $y$. Since $y \in \operatorname{Hull}(\sigma) \subset \operatorname{Hull}\left(\Gamma_{X}(y)\right)$, it follows that $y$ is a critical point of the distance function to $X$, which concludes the proof.
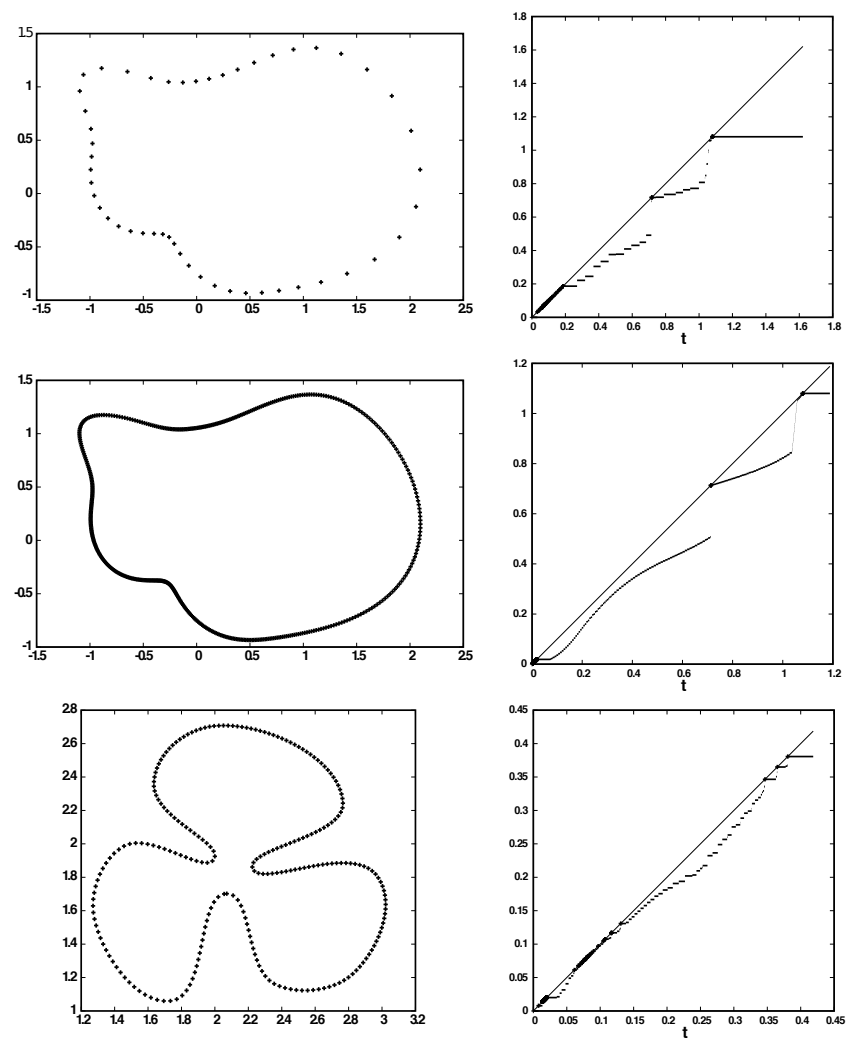

Figure 5: Various point sets $P$ on the left and corresponding convexity defects function $c_{P}$ on the right.

\subsection{Sampling conditions based on convexity de- fects functions}

We assemble the pieces and deduce conditions under which the Čech complex and the Rips complex of a finite set of points retrieve the topology of the shape the points sample. Throughout the section, $X$ designates a compact subset of $\mathbb{R}^{n}$ and $P$ is a finite set of points, whose Hausdorff distance to $X$ is $\varepsilon$ or less. Reconstruction results will relie on the stability of $h_{X}$ under perturbations of $X$ (see Figure 5) which is a consequence of the 1-Lipschitz property of the map $\sigma \mapsto \operatorname{Rad}(\sigma)$ established in Lemma 8 in the appendix.
LEMMA 4. For every pair of subsets $X$ and $P$ of $\mathbb{R}^{n}$ such that $d_{H}(X, P) \leq \varepsilon$ and for every $t \geq 0$, we have

$$
h_{P}(t) \leq h_{X}(t+\varepsilon)+2 \varepsilon .
$$

Proof. Consider a non-empty subset $\sigma \subset P$ with $\operatorname{Rad}(\sigma) \leq$ $t$ and set $\xi=X \cap \sigma^{\varepsilon}$. By construction, $\xi$ is non-empty and $d_{H}(\xi, \sigma) \leq \varepsilon$. Hence, Lemma 8 implies that $\operatorname{Rad}(\xi) \leq t+\varepsilon$. Using $\operatorname{Hull}\left(\xi^{\varepsilon}\right)=\operatorname{Hull}(\xi)^{\varepsilon}$, we get that $\operatorname{Hull}(\sigma) \subset \operatorname{Hull}(\xi)^{\varepsilon} \subset$ $X^{h_{X}(t+\varepsilon)+\varepsilon} \subset P^{h_{X}(t+\varepsilon)+2 \varepsilon}$, yielding the result.

\section{Reconstruction with the Čech complex.}

The assumption that $d_{H}(X, P) \leq \varepsilon$ implies the following chain of inclusions: $P^{\alpha} \subset X^{\alpha+\varepsilon} \subset P^{\alpha+2 \varepsilon} \subset X^{\alpha+3 \varepsilon}$. From [7], we know that whenever we consider four nested spaces $P_{0} \subset X_{0} \subset$ $P_{1} \subset X_{1}$ such that $X_{1}$ deformation retracts to $X_{0}$ and $P_{1}$ deformation retracts to $P_{0}$, then $X_{0}$ deformation retracts to $P_{0}$. Applying this result to our context combined with the Isotopy Theorem and the characterization of critical points given in Lemma 3 , we deduce immediately that $X^{\alpha+\varepsilon}$ deformation retracts to $P^{\alpha}$ whenever the following two conditions are fulfilled:

$$
\begin{array}{ll}
h_{X}(t)<t, & \forall t \in[\alpha+\varepsilon, \alpha+3 \varepsilon], \\
h_{P}(t)<t, & \forall t \in[\alpha, \alpha+2 \varepsilon] .
\end{array}
$$

Since $d_{H}(X, P) \leq \varepsilon$, Lemma 4 implies that $h_{P}(t) \leq h_{X}(t+$ $\varepsilon)+2 \varepsilon$ and therefore the above two conditions are fulfilled as soon as the following stronger condition holds: $h_{X}(t)<t-3 \varepsilon$, $\forall t \in[\alpha+\varepsilon, \alpha+3 \varepsilon]$. Because $h_{X}$ is non-negative, this condition implies that $2 \varepsilon<\alpha$. Because $h_{X}$ is increasing, it also implies that $h_{X}(t)<t$ for all $t \in[\alpha-2 \varepsilon, \alpha+3 \varepsilon]$, showing that $t$-offsets of $X$ in the interval $[\alpha-2 \varepsilon, \alpha+3 \varepsilon]$ are all homotopy equivalent. We summarize our findings in the following theorem:

THEOREM 4. Let $\varepsilon, \alpha>0$ such that $2 \varepsilon<\alpha$. Let $P$ be a finite set of points whose Hausdorff distance to a compact subset $X$ is $\varepsilon$ or less. The Čech complex $\mathcal{C}(P, \alpha)$ is homotopy equivalent to the $(\alpha-2 \varepsilon)$-offset of $X$ whenever $h_{X}(t)<t-3 \varepsilon$ for all $t \in$ $[\alpha+\varepsilon, \alpha+3 \varepsilon]$.

\section{Reconstruction with the Rips complex.}

If furthermore we suppose that $c_{P}\left(\vartheta_{n} \beta\right)<2 \alpha-\vartheta_{n} \beta$, we can apply Theorem 1 and deduce that $(\alpha, \beta)$-almost Rips complexes of $P$ deformation retracts to the Cech complex $\mathcal{C}(P, \alpha)$. Using Lemma 4 we get that $c_{P}\left(\vartheta_{n} \beta\right) \leq h_{P}\left(\vartheta_{n} \beta\right) \leq h_{X}\left(\vartheta_{n} \beta+\varepsilon\right)+2 \varepsilon$ and the hypothesis of Theorem 1 is fulfilled whenever $h_{X}\left(\vartheta_{n} \beta+\right.$ $\varepsilon)<2 \alpha-\vartheta_{n} \beta-2 \varepsilon$. Because $h_{X}$ is non-negative, this condition implies that $2 \varepsilon<2 \alpha-\vartheta_{n} \beta$. Because $h_{X}$ is increasing, it also implies that $h_{X}(t)<t-3 \varepsilon, \forall t \in[\alpha+\varepsilon, \alpha+3 \varepsilon]$ and the hypothesis of Theorem 4 is also fulfilled. We deduce the following theorem:

THEOREM 5. Let $\varepsilon, \alpha$ and $\beta$ be three non-negative real numbers such that $\alpha \leq \beta$ and $\eta=2 \alpha-\vartheta_{n} \beta-2 \varepsilon>0$. Let $P$ be $a$ finite set of points whose Hausdorff distance to a compact subset $X$ is $\varepsilon$ or less. Then, any $(\alpha, \beta)$-almost Rips complex of $P$ is homotopy equivalent to the $\eta$-offset of $X$ whenever $\alpha$ is an inert value of $P$ and $h_{X}\left(\vartheta_{n} \beta+\varepsilon\right)<2 \alpha-\vartheta_{n} \beta-2 \varepsilon$.

\subsection{Connections with the critical function}

In this section, we show that the class of shapes with an upper bounded convexity defect function are equivalent to the class of shapes with a lower bounded critical function. To make this idea 


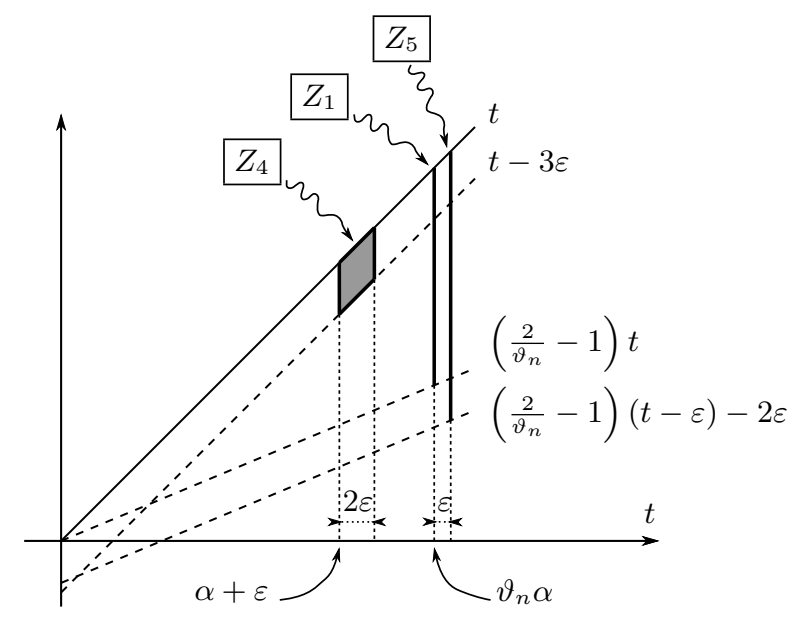

Figure 6: For $i \in\left\{\begin{array}{l|l|l|l}1 & 4 & 5\end{array}\right\}$, the hypotheses of Theorem $i$ are depicted as regions $Z_{i}$ avoided by the graph of a convexity defects function. Specifically, if $c_{P} \cap Z_{1}=\emptyset$, Theorem 1 implies $\mathcal{R}(P, \alpha) \simeq P^{\alpha}$. If $h_{X} \cap Z_{4}=\emptyset$, Theorem 4 implies $\mathcal{C}(P, \alpha) \simeq X^{\alpha-2 \varepsilon}$. If $h_{X} \cap Z_{5}=\emptyset$, Theorem 5 implies $\mathcal{R}(P, \alpha) \simeq X^{2 \alpha-\vartheta_{n} \alpha-2 \varepsilon}$.

precise, we need to recall the definition of critical functions instrumental in expressing sampling conditions for a larger class of objects than shapes with a positive reach in [15]. Even though the distance function to $X$ is not differentiable, it is possible to define a generalized gradient function $\nabla_{X}: \mathbb{R}^{n} \backslash X \rightarrow \mathbb{R}$ that coincides with the usual gradient at points where $d(\cdot, X)$ is differentiable and that vanishes precisely at points that are critical [15]. Specifically,

$$
\nabla_{X}(y)=\frac{y-\operatorname{Center}\left(\Gamma_{X}(y)\right)}{d(y, X)} .
$$

The critical function $\chi_{X}: \mathbb{R}_{+}^{*} \rightarrow \mathbb{R}_{+}$is defined by

$$
\chi_{X}(t)=\inf _{d(y, X)=t}\|\nabla(y)\| .
$$

For $0<\mu \leq 1$, authors in $[15]$ define the $\mu$-reach of $X$ as $r_{\mu}(X)=$ inf $\left\{t>0, \chi_{X}(t)<\mu\right\}$. The terminology comes from the fact that $r_{1}(X)$ coincides with the usual reach of $X$. Observe that $r_{\mu}(X) \geq R$ is equivalent to $\chi_{X}(t) \geq \mu$ for all $t \in(0, R)$. Our first lemma provides a lower bound on $\chi_{X}$ at $t$, assuming an upper bound on $c_{X}$ at $t$.

LEMMA 5. For all compact set $X \subset \mathbb{R}^{n}$, all $0 \leq \mu \leq 1$ and all $t \geq 0$, the following implication holds:

$$
c_{X}(t)<(1-\mu) t \quad \Longrightarrow \quad \chi_{X}(t)>\mu \text {. }
$$

Proof. Consider $y \in \mathbb{R}^{n}$ whose distance to $X$ is $t$ and let us prove that $\left\|\nabla_{X}(y)\right\|>\mu$. Let $\sigma=\Gamma_{X}(y)$ be the set of points in $X$ with minimum distance to $y$. Suppose the smallest ball enclosing $\sigma$ has center $z$ and radius $s$. Since $s \leq t$, we get $c_{X}(s) \leq c_{X}(t)<$ $(1-\mu) t$ and therefore $t-\|y-z\| \leq \bar{d}(z, X) \leq c_{X}(s)<(1-\mu) t$. It follows that $\left\|\nabla_{X}(y)\right\|=\frac{\|z-y\|}{t}>\mu$.

Next lemma can be thought of as a converse of the previous lemma, since it provides an upper bound on $h_{X}$ over the interval $[0, R]$, assuming a lower bound on the critical function $\chi_{X}$ over the interval $(0, R)$. It extends a result in $|7|$ and says intuitively that the convex hull of point set $\sigma \subset X$ cannot be too far away from a shape $X$, assuming $\sigma$ can be enclosed in a ball of small radius $t$ and $X$ has a positive $\mu$-reach.
Lemma 6. Consider two real numbers $\mu \in(0,1]$ and $R \geq 0$. Let $X \subset \mathbb{R}^{n}$ be a compact set such that $\chi_{X}(t) \geq \mu$ for all $t \in$ $(0, R)$. Then, for all $0 \leq t \leq R$, one has:

$$
h_{X}(t) \leq \frac{1+\mu(1-\mu)-\sqrt{1-\mu(2-\mu)\left(\frac{t}{R}\right)^{2}}}{\mu(2-\mu)} R .
$$

Proof. Given $\sigma \subset X$ with $\operatorname{Rad}(\sigma) \leq R$ and $y_{0} \in \operatorname{Hull}(\sigma)$, we establish an upper bound on $d\left(y_{0}, X\right)$ expressed as a function of $\operatorname{Rad}(\sigma)$. Consider an integral line $\mathcal{C}_{y_{0}}$ of the flow associated to the distance function to $X$ and starting at point $y_{0}[30,16]$. Suppose this integral line is parameterized by arc length and set $y_{s}=\mathcal{C}_{y_{0}}(s)$. For $s<R-d\left(y_{0}, X\right)$, one has $d\left(y_{s}, X\right) \leq$ $d\left(y_{0}, X\right)+s<R$ and therefore $\chi_{X}\left(d\left(y_{s}, X\right)\right) \geq \mu$ which implies $\left\|\nabla_{X}\left(y_{s}\right)\right\| \geq \mu$. In particular, the integral line $\mathcal{C}_{y_{0}}$ does not reach any critical point as long as $s<R-d\left(y_{0}, X\right)$ and $\mathcal{C}_{y_{0}}$ can at least be parameterized on the interval $\left[0, R-d\left(y_{0}, X\right)\right]$. Since the norm of the gradient $\left\|\nabla_{X}\left(y_{s}\right)\right\| \geq \mu$ is equal to the right derivative of $s \mapsto d\left(y_{s}, X\right)$ (see [30, 16]), we obtain that

$$
\frac{d\left(y_{s}, X\right)-d\left(y_{0}, X\right)}{s} \geq \mu \text {. }
$$

Applying Lemma 1 with $x=y_{s}$ and $y=y_{0}$ gives $d\left(y_{s}, X\right)^{2} \leq$ $d\left(y_{s}, \sigma\right)^{2} \leq s^{2}+\operatorname{Rad}(\sigma)^{2}$ from which we deduce the inequality $\left(d\left(y_{0}, X\right)+\mu s\right)^{2} \leq s^{2}+\operatorname{Rad}(\sigma)^{2}$. Plugging $s=R-d\left(y_{0}, X\right)$, setting $\delta=\frac{d\left(y_{0}, X\right)}{R}, \rho=\frac{\operatorname{Rad}(\sigma)}{R}$ and rearranging this inequality gives us

$$
\mu(2-\mu) \delta^{2}-2\left(1+\mu-\mu^{2}\right) \delta+1-\mu^{2}+\rho^{2} \geq 0 .
$$

Since $\delta \leq 1$ we get $\delta \leq \frac{1+\mu(1-\mu)-\sqrt{1-\rho^{2} \mu(2-\mu)}}{\mu(2-\mu)}$, yielding the result.

The upper bound on $h_{X}$ is an arc of ellipse which tends to an arc of parabola as $\mu \rightarrow 0$; see Figure 7. Note that since $h_{X}(t) \leq t$ for all $t$, this upper bound is only relevant when under the diagonal. For $\mu=1$, we get $h_{X}(t) \leq R-\sqrt{R^{2}-t^{2}}$ as in [7]. Equivalently, the graph of $h_{X}$ is below the circle with radius $R$ and center $(0, R)$.

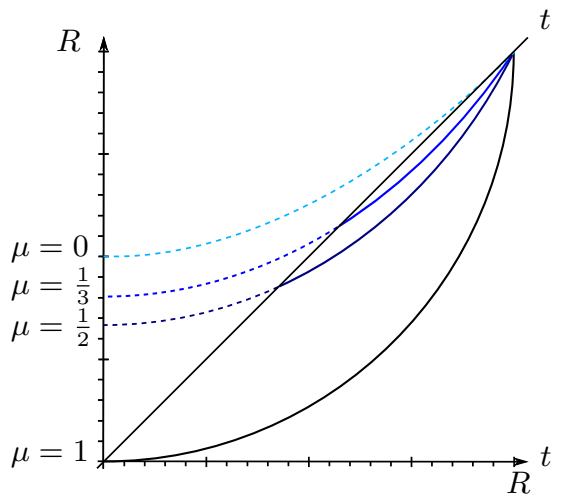

Figure 7: Upper bound on $h_{X}$ for $\mu \in\left\{0, \frac{1}{3}, \frac{1}{2}, 1\right\}$ provided by Lemma 6

\subsection{Reconstructing shapes with a positive $\mu$ - reach}

Given a shape $X$ whose $\mu$-reach $R$ is positive and a finite point set $P$ such that $d_{H}(P, X) \leq \varepsilon$, we compute the largest value of the ratio $\frac{\varepsilon}{R}$ for which the Cech complex $\mathcal{C}(P, \alpha)$ or the Rips complex $\mathcal{R}(P, \alpha)$ provide a topologically correct reconstruction of $X$ for 

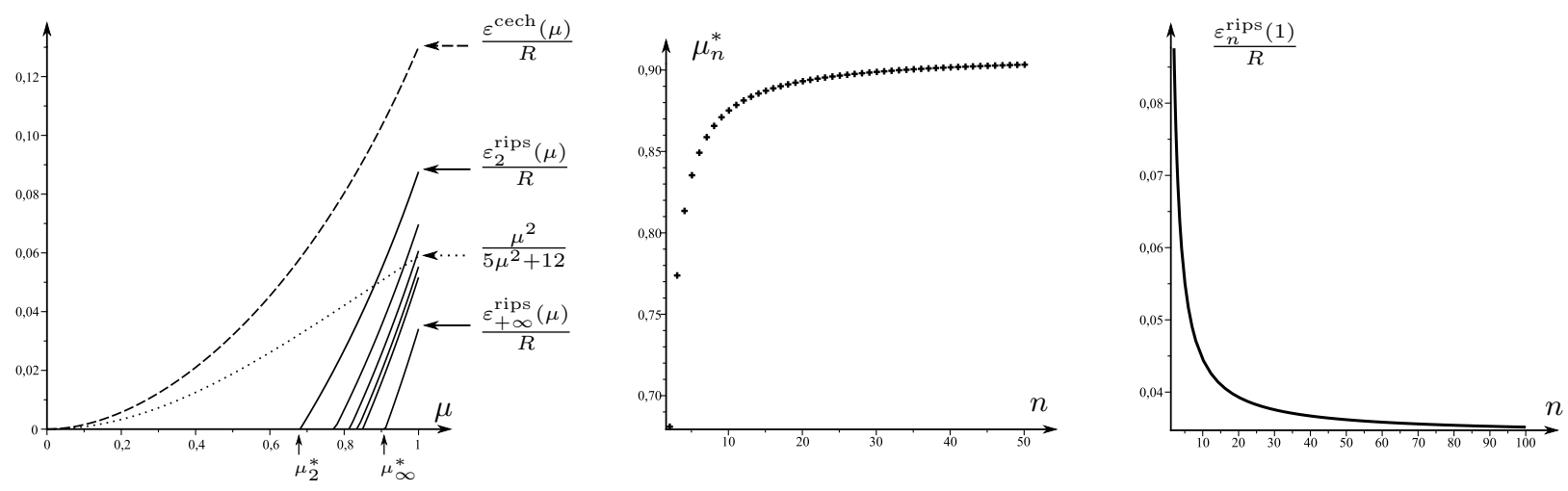

Figure 8: Left: Best ratios $\frac{\varepsilon}{R}$ we can get for a correct reconstruction of a shape with a positive $\mu$-reach either with the Čech complex or the Rips complex for $n \in\{2,3,4,5,6,+\infty\}$; comparison with the ratio obtained in [15]. Middle: $\mu_{n}^{*}$ as a function of $n$. Right: $\frac{\varepsilon_{n}^{\text {rips }}(1)}{R}$ as a function of $n$.

a suitable value of the parameter $\alpha$. Computations were realized using a computer algebra system and details are skipped.

\section{Reconstruction with the Čech complex.}

Combining Theorem 4 and Lemma 6 , we obtain that the Čech complex $\mathcal{C}(P, \alpha)$ is homotopy equivalent to $X^{\alpha-2 \varepsilon}$ for all $\alpha \in$ $(2 \varepsilon, R-3 \varepsilon]$ whenever the following inequality holds for all $t \in$ $[\alpha+\varepsilon, \alpha+3 \varepsilon]$ :

$$
\frac{1+\mu(1-\mu)-\sqrt{1-\mu(2-\mu)\left(\frac{t}{R}\right)^{2}}}{\mu(2-\mu)} R<t-3 \varepsilon .
$$

Eliminating the square root, we can replace the above inequality by $H<0$ where $H$ is a polynomial of degree 2 in $t$. It follows that the above condition holds whenever the absolute difference between the two roots $\lambda_{1}, \lambda_{2}$ of $H$ is greater than $2 \varepsilon$. The condition $\left|\lambda_{2}-\lambda_{1}\right|>2 \varepsilon$ can be rewrote as the positivity of a polynomial of degree 2 in $\varepsilon$ and holds whenever $\varepsilon$ is smaller than the greatest root $\varepsilon^{\text {cech }}(\mu)$ whose value divided by $R$ is:

$\frac{-3 \mu+3 \mu^{2}-3+\sqrt{-8 \mu^{2}+4 \mu^{3}+18 \mu+2 \mu^{4}+9+\mu^{6}-4 \mu^{5}}}{-7 \mu^{2}+22 \mu+\mu^{4}-4 \mu^{3}+1}$.

Interestingly, $\varepsilon^{\text {cech }}(\mu)$ does not depend on the ambient dimension $n$. Plotting the ratio $\frac{\varepsilon^{\operatorname{cech}}(\mu)}{R}$ as a function of $\mu$ (see Figure 8 left), we observe that it is positive for all $\mu \in(0,1]$ and improves on the upper bound $\frac{\mu^{2}}{5 \mu^{2}+12}$ established in $|15|$. Still, for $\mu=1$, we get $\frac{\varepsilon^{\text {cech }}(1)}{R}=\frac{-3+\sqrt{22}}{13} \approx 0.13$ which is not as good as the value $3-\sqrt{8} \approx 0.17$ obtained in 32

\section{Reconstruction with the Rips complex.}

Combining Theorem 5 with $\beta=\alpha$ and Lemma 6 we get that the Rips complex $\mathcal{R}(P, \alpha)$ is homotopy equivalent to $X^{2 \alpha-\vartheta_{n} \alpha-2 \varepsilon}$ for all $\alpha \in\left(\frac{2 \varepsilon}{2-\vartheta_{n}}, \frac{R-\varepsilon}{\vartheta_{n}}\right]$ whenever

$$
\frac{1+\mu(1-\mu)-\sqrt{1-\mu(2-\mu)\left(\frac{\vartheta_{n} \alpha+\varepsilon}{R}\right)^{2}}}{\mu(2-\mu)} R<2 \alpha-\vartheta_{n} \alpha-2 \varepsilon .
$$

As before, we can eliminate the square root, replacing the above inequality by $H<0$ where $H$ is a polynomial of degre 2 in $\varepsilon$ and $\alpha$. Since we are looking for the greatest value of $\varepsilon$ for which $H<0$, we may assume that $\frac{\partial H}{\partial \alpha}=0$. Plugging the value of $\alpha$ for which $\frac{\partial H}{\partial \alpha}=0$ in $H$, we get a polynomial of degree 2 in $\varepsilon$ whose greatest root $\varepsilon_{n}^{\text {rips }}(\mu)$ gives the supremum of $\varepsilon$ for which the above inequality holds. Plotting the ratio $\frac{\varepsilon_{n}^{\text {rips }}(\mu)}{R}$ as a function of $\mu$, we observe that the ratio is only positive on a subinterval $\left(\mu_{n}^{*}, 1\right]$ of $(0,1]$; see Figure 8 left. Hence, we can only guarantee that Rips complexes provide a correct reconstruction for shapes with a positive $\mu$-reach when $\mu>\mu_{n}^{*}$. In Figure 8 . middle, we plotted $\mu_{n}^{*}$ as a function of $n . \mu_{n}^{*}$ increases with $n$ and we were able to prove using a computer algebra system that $\mu_{n}^{*}$ tends to $\sqrt{2 \sqrt{2}-2} \approx 0.91$ as $n \rightarrow+\infty$. In Figure 8 right, we plotted $\frac{\varepsilon_{n}^{\text {rips }}(1)}{R}$ as a function of $n$. For a fixed $R, \varepsilon_{n}^{\text {rips }}(1)$ decreases with $n$ and similarly, we proved that $\lim _{n \rightarrow+\infty} \varepsilon_{n}^{\mathrm{rips}}(1)=\frac{2 \sqrt{2-\sqrt{2}}-\sqrt{2}}{2+\sqrt{2}} R \approx 0.034 R$.

\section{CONCLUSION}

A glance at the proof of our main result (Theorem 1) reveals the centrality of Jung's Theorem that relates the diameter and radius of any subset of an Euclidean space. Observe that in $L^{\infty}$ spaces the diameter of a set is always twice its radius. Thus, Čech and Rips complexes coincide and Theorem 1 degenerates into a trivial form. The definition of our function $c_{X}$ is purely metric. The definition of $h_{X}$ only requires, beside a metric, a notion of (local) convex hull which can be defined from geodesics for a large class of metric spaces (see the definition of complete length spaces in [26]). A natural question for future work is whether our results extend to more general metric spaces such as Riemannian manifolds, $L^{p}$ spaces or abstract metric spaces for which an analog of Jung's Theorem would be replaced by an axiom.

Lemma 10 says that the hypotheses of Theorem 1 are stable under small metric perturbations. In particular, our relaxed definition of almost Rips complexes (unlike the usual notion of Rips complexes) should allow us to apply Theorem 1 in the context of Gromov-Hausdorff distances.

Another natural extension would be to consider local measures of convexity defects and define sampling densities adapted to the local geometry of the sampled set in the spirit of [2].

\section{REFERENCES}

[1] N. Amenta, D. Attali, and O. Devillers. Complexity of Delaunay triangulation for points on lower-dimensional polyhedra. In Proc. ACM-SIAM Sympos. Discrete Algorithms 
(SODA'07), New-Orleans, Lousiana, USA, January 7-9 2007.

[2] N. Amenta and M. Bern. Surface reconstruction by Voronoi filtering. Discrete and Computational Geometry, 22(4):481-504, 1999.

[3] N. Amenta, M. Bern, and D. Eppstein. The crust and the $\beta$-skeleton: Combinatorial curve reconstruction. Graphical Models and Image Processing, 60(2):125-135, 1998.

[4] D. Attali. $r$-regular shape reconstruction from unorganized points. Computational Geometry: Theory and Applications, 10:239-247, 1998.

[5] D. Attali and J.-D. Boissonnat. A linear bound on the complexity of the Delaunay triangulation of points on polyhedral surfaces. Discrete and Computational Geometry, 31(3):369-384, February 2004.

[6] D. Attali, J.-D. Boissonnat, and A. Lieutier. Complexity of the Delaunay triangulation of points on surfaces: the smooth case. In 19th ACM Symposium on Computational Geometry, pages 201-210, San-Diego, California, USA, June 2003.

[7] D. Attali and A. Lieutier. Reconstructing shapes with guarantees by unions of convex sets. In 26th Ann. Sympos. Comput. Geom., Snowbird, Utah, 2010. download. full-version.

[8] D. Attali, A. Lieutier, and D. Salinas. Efficient Data Structure for Representing and Simplifying Simplicial Complexes in High Dimension. In Proc. 27th Ann. Sympos. Comput. Geom., Paris, France, June 13-15 2011.

[9] J. A. Barmak and E. G. Minian. Strong homotopy types, nerves and collapses. Technical report, arXiv:0907.2954v1, 2009.

[10] A. Bjorner. Topological methods. In Handbook of combinatorics (vol. 2), page 1850. MIT Press, 1996.

[11] J. Boissonnat and F. Cazals. Smooth surface reconstruction via natural neighbour interpolation of distance functions. Computational Geometry: Theory and Applications, 22(1-3):185-203, 2002.

[12] J. Boissonnat, O. Devillers, and S. Hornus. Incremental construction of the Delaunay triangulation and the Delaunay graph in medium dimension. In Proceedings of the 25th annual symposium on Computational geometry, pages 208-216. ACM, 2009.

[13] J. Boissonnat and A. Ghosh. Manifold reconstruction using tangential Delaunay complexes. In Proceedings of the 2010 annual symposium on Computational geometry, pages 324-333. ACM, 2010.

[14] E. Chambers, V. de Silva, J. Erickson, and R. Ghrist. Vietoris-rips complexes of planar point sets. Discrete and Computational Geometry, pages 1-16, 2010.

[15] F. Chazal, D. Cohen-Steiner, and A. Lieutier. A sampling theory for compact sets in Euclidean space. Discrete and Computational Geometry, 41(3):461-479, 2009.

[16] F. Chazal and A. Lieutier. The $\lambda$-medial axis. Graphical Models, 67(4):304-331, 2005.

[17] F. Chazal and A. Lieutier. Smooth Manifold Reconstruction from Noisy and Non Uniform Approximation with Guarantees. Computational Geometry: Theory and Applications, 40:156-170, 2008.

[18] F. Chazal and S. Oudot. Towards persistence-based reconstruction in euclidean spaces. In Proc. ACM Symposium on Computational Geometry, pages 232-241, 2008.
[19] V. De Silva. A weak characterisation of the Delaunay triangulation. Geometriae Dedicata, 135(1):39-64, 2008.

[20] V. de Silva and R. Ghrist. Coverage in sensor networks via persistent homology. Algebraic \& Geometric Topology, 7:339-358, 2007.

[21] T. Dey. Curve and surface reconstruction: algorithms with mathematical analysis. Cambridge Univ Pr, 2007.

[22] T. K. Dey, H. Edelsbrunner, and S. Guha. Computational topology. In B. Chazelle, J. E. Goodman, and R. Pollack, editors, Advances in Discrete and Computational Geometry, volume 223 of Contemporary Mathematics. AMS, Providence, 1999.

[23] H. Edelsbrunner. The union of balls and its dual shape. In Proceedings of the ninth annual symposium on Computational geometry, pages 218-231. ACM, 1993.

[24] H. Edelsbrunner and E. M "ucke. Three-dimensional alpha shapes. In Proceedings of the 1992 workshop on Volume visualization, pages 75-82. ACM, 1992.

[25] H. Edelsbrunner and N. Shah. Triangulating topological spaces. In International Journal of Computational Geometry and Applications, volume 7, pages 365-378. Citeseer, 1997.

[26] M. Gromov, M. Katz, P. Pansu, and S. Semmes. Metric structures for Riemannian and non-Riemannian spaces. Birkhauser, 2006.

[27] K. Grove. Critical point theory for distance functions. In Proc. of Symposia in Pure Mathematics, volume 54, pages 357-386, 1993.

[28] J. Hausmann. On the Vietoris-Rips Complexes and a Cohomology Theory for Metric Spaces. In Prospects in topology: proceedings of a conference in honor of William Browder, page 175. Princeton Univ Pr, 1995.

[29] J. Latschev. Vietoris-Rips complexes of metric spaces near a closed Riemannian manifold. Archiv der Mathematik, 77(6):522-528, 2001.

[30] A. Lieutier. Any open bounded subset of $\mathbb{R}^{n}$ has the same homotopy type as its medial axis. Computer-Aided Design, 36(11):1029-1046, 2004.

[31] J. Munkres. Elements of algebraic topology. Perseus Books, 1993.

[32] P. Niyogi, S. Smale, and S. Weinberger. Finding the Homology of Submanifolds with High Confidence from Random Samples. Discrete Computational Geometry, 39(1-3):419-441, 2008.

[33] E. Welzl. Smallest enclosing disks (balls and ellipsoids). In H. Maurer, editor, New Results and New Trends in Computer Science, volume 555, pages 359-370. Springer, 1991.

[34] A. Zomorodian. Fast construction of the Vietoris-Rips complex. Computers \& Graphics, 2010.

[35] A. Zomorodian. The tidy set: a minimal simplicial set for computing homology of clique complexes. In Proceedings of ACM symposium of computational geometry, 2010.

\section{APPENDIX}

In this appendix, we review useful results on smallest enclosing balls and establish the stability of hypotheses of Theorem 1 under small perturbations of the point set $P$.

LEMMA 7. The smallest ball enclosing a non-empty bounded set of $\mathbb{R}^{n}$ exists and is unique.

ProOF. Let $\sigma$ be a non-empty bounded set of $\mathbb{R}^{n}$. We first es- 
tablish the existence of a smallest ball enclosing $\sigma$. Given a point $y \in \mathbb{R}^{n}$ and a real number $s \geq 0$, we first prove that the set $\mathcal{B}(y, s)$ of closed balls passing through $y$ and with radius $s$ or less is compact. Indeed, representing a closed ball with center $z$ and radius $r$ by point $(z, r)$ in $\mathbb{R}^{n+1}$, we can write

$$
\mathcal{B}(y, s)=\left\{(z, r) \in \mathbb{R}^{n+1} \mid\|z-y\| \leq r \leq s\right\},
$$

which is closed by definition and bounded since for all balls $\left(z_{0}, r_{0}\right)$ and $\left(z_{1}, r_{1}\right)$ in $\mathcal{B}(y, s)$, we have $\left\|z_{0}-z_{1}\right\|+\left|r_{0}-r_{1}\right| \leq 3 s$. The set of closed balls containing $\sigma$ and whose radii are smaller than or equal to the diameter of $\sigma$ is

$$
\mathcal{B}(\sigma)=\bigcap_{y \in \sigma} \mathcal{B}(y, \operatorname{Diam}(\sigma)) .
$$

This set is non-empty and compact and therefore, the continuous map $(z, r) \mapsto r$ on $\mathcal{B}(\sigma)$ is bounded below and attains its infimum. The uniqueness is easy to establish by contradiction, as explained in [33].

Next lemma states that the radius and center of the smallest enclosing ball are stable.

LEMMA 8. For every non-empty bounded subsets $\sigma$ and $\sigma^{\prime}$ of $\mathbb{R}^{n}$ such that $d_{H}\left(\sigma, \sigma^{\prime}\right) \leq \varepsilon$, we have $\left|\operatorname{Rad}(\sigma)-\operatorname{Rad}\left(\sigma^{\prime}\right)\right| \leq \varepsilon$ and $\left\|\operatorname{Center}(\sigma)-\operatorname{Center}\left(\sigma^{\prime}\right)\right\| \leq \sqrt{2 \varepsilon \operatorname{Rad}(\sigma)+\varepsilon^{2}}$.

Proof. Writing $B$ for the smallest ball enclosing $\sigma$, we have $\sigma^{\prime} \subset \sigma^{\varepsilon} \subset B^{\varepsilon}$, showing that $\operatorname{Rad}\left(\sigma^{\prime}\right) \leq \operatorname{Rad}(\sigma)+\varepsilon$.

For the second part of the lemma, set $z=\operatorname{Center}(\sigma), z^{\prime}=$ $\operatorname{Center}\left(\sigma^{\prime}\right), r=\operatorname{Rad}(\sigma)$ and $r^{\prime}=\operatorname{Rad}\left(\sigma^{\prime}\right)$. Suppose $z \neq z^{\prime}$ for otherwise the result is clear and consider the hyperplane $H$ passing through $z$ and orthogonal to the segment $z z^{\prime}$. Let $\xi=\bar{\sigma} \cap \partial B$. By construction, $\xi$ is closed and has the same smallest enclosing ball as $\sigma$. Thus, $z \subset \operatorname{Hull}(\xi)$ and the closed half-space $H^{+}$that $H$ bounds and which avoids $z^{\prime}$ intersects $\xi$. Let $p$ be a point in this intersection. By choice of $p$ in $H^{+}$, the triangle $p z z^{\prime}$ is obtuse at vertex $z$ and $\left\|z^{\prime}-p\right\|^{2} \geq\|z-p\|^{2}+\left\|z^{\prime}-z\right\|^{2}$. Using $\|z-p\|=r$ and $\left\|z^{\prime}-p\right\| \leq r^{\prime}+\varepsilon$ we obtain $\left\|z^{\prime}-z\right\|^{2} \leq\left(r^{\prime}+\varepsilon\right)^{2}-r^{2}$. Interchanging the roles of $\sigma$ and $\sigma^{\prime}$ yields:

$$
\left\|z^{\prime}-z\right\|^{2} \leq \min \left\{\left(r^{\prime}+\varepsilon\right)^{2}-r^{2},(r+\varepsilon)^{2}-r^{\prime 2}\right\} .
$$

Note that $\left(r^{\prime}+\varepsilon\right)^{2}-r^{2} \leq(r+\varepsilon)^{2}-r^{\prime 2}$ if and only if $r \geq r^{\prime}$. Considering in turn each of the two cases $r \leq r^{\prime}$ and $r^{\prime} \leq r$, we get the desired inequality.

Next lemma states the stability of $c_{X}$ under perturbations of $X$. The stability of $h_{X}$ is established in Lemma 4

LEMMA 9. For every pair of subsets $X$ and $Y$ of $\mathbb{R}^{n}$ such that $d_{H}(X, Y) \leq \varepsilon$ and for every $t \geq 0$, we have

$$
c_{Y}(t) \leq c_{X}(t+\varepsilon)+\sqrt{2 t \varepsilon+\varepsilon^{2}}+\varepsilon .
$$

Proof. Consider a non-empty subset $\sigma \subset Y$ with $\operatorname{Rad}(\sigma) \leq$ $t$ and set $\xi=X \cap \sigma^{\varepsilon}$. By construction, $\xi$ is non-empty and $d_{H}(\xi, \sigma) \leq \varepsilon$. Hence, setting $\delta=\sqrt{2 t \varepsilon+\varepsilon^{2}}$, Lemma 8 implies that $\|\operatorname{Center}(\sigma)-\operatorname{Center}(\xi)\| \leq \delta$, we get

$\operatorname{Center}(\sigma) \subset \operatorname{Center}(\xi)^{\delta} \subset X^{h_{X}(t+\varepsilon)+\delta} \subset Y^{h_{X}(t+\varepsilon)+\delta+\varepsilon}$, yielding to the result.
Given a point set $P \subset \mathbb{R}^{n}$, we say that a map $f: P \rightarrow \mathbb{R}^{n}$ is an $\varepsilon$-small perturbation of $P$ if $f$ is injective and $\|p-f(p)\| \leq \varepsilon$ for all points $p \in P$. Given a simplicial complex $K$, we define the simplicial complex $f(K)=\{f(\sigma) \mid \sigma \in K\}$.

LeMma 10. Let $P \subset \mathbb{R}^{n}$ be a finite set of points. Consider two real numbers $\beta \geq \alpha \geq 0$ such that

$$
c_{P}\left(\vartheta_{\mathrm{n}} \beta\right)<2 \alpha-\vartheta_{\mathrm{n}} \beta
$$

and suppose moreover that $\alpha$ is an inert value of $P$. Then, there exist $\varepsilon>0$ and $\beta^{\prime}>\beta$ such that for all $\varepsilon$-small perturbations $f$ of $P$, we have:

(i) $c_{f(P)}\left(\vartheta_{\mathrm{n}} \beta^{\prime}\right)<2 \alpha-\vartheta_{\mathrm{n}} \beta^{\prime}$;

(ii) $\mathcal{C}(f(P), \alpha)=f(\mathcal{C}(P, \alpha))$;

(iii) if Flag $G$ is an $(\alpha, \beta)$-almost Rips complex of $P$, then Flag $f(G)$ is an $\left(\alpha, \beta^{\prime}\right)$-almost Rips complex of $f(P)$.

Proof. Let us establish $(i)$. For this, set $t=\vartheta_{\mathrm{n}} \beta$ and define $\bar{t}=\min \{\operatorname{Rad}(\sigma) \mid \emptyset \neq \sigma \subset P$ and $\operatorname{Rad}(\sigma)>t\}$. By construction, $\bar{t}>t$. Lemma 9 ensures that for all subsets $P^{\prime} \subset \mathbb{R}^{n}$ within Hausdorff distance $\varepsilon$ from $P$ and for all $t^{\prime} \geq 0$, the following implication holds:

$$
c_{P^{\prime}}\left(t^{\prime}\right)<c_{P}\left(t^{\prime}+\varepsilon\right)+\sqrt{2 t^{\prime} \varepsilon+\varepsilon^{2}}+\varepsilon .
$$

By assumption, we have $2 \alpha-t-c_{P}(t)>0$. By choosing $\varepsilon>0$ small enough, we can always find $t^{\prime}>t$ such that (1) $t^{\prime}+\varepsilon<\bar{t}$, (2) $2 \alpha-t^{\prime}-c_{P}(t)>0$ and (3) $\sqrt{2 t^{\prime} \varepsilon+\varepsilon^{2}}+\varepsilon \leq \frac{2 \alpha-t^{\prime}-c_{P}(t)}{2}$. Since $c_{P}\left(t^{\prime}+\varepsilon\right)=c_{P}(t)$, it follows that

$$
c_{P^{\prime}}\left(t^{\prime}\right)<c_{P}(t)+\frac{2 \alpha-t^{\prime}-c_{P}(t)}{2}<2 \alpha-t^{\prime}
$$

and $(i)$ is proved with $\beta^{\prime}=t^{\prime} / \vartheta_{\mathrm{n}}$. By choosing $\varepsilon>0$ small enough, we can always assume that in addition to conditions (1), (2) and (3), we have (4) $\varepsilon<\beta^{\prime}-\beta$ and (5) $\operatorname{Rad}(\sigma) \notin[\alpha-\varepsilon, \alpha+\varepsilon]$ for all $\emptyset \neq \sigma \subset P$. Let $f$ be an $\varepsilon$-small perturbation of $P$. Using Lemma 8 and condition (5), we get

$$
\begin{array}{r}
\sigma \in \mathcal{C}(P, \alpha) \Leftrightarrow \operatorname{Rad}(\sigma) \leq \alpha \Leftrightarrow \operatorname{Rad}(\sigma) \leq \alpha-\varepsilon \\
\Rightarrow \operatorname{Rad}(f(\sigma)) \leq \alpha \Leftrightarrow f(\sigma) \in \mathcal{C}(f(P), \alpha)
\end{array}
$$

and

$$
\begin{array}{r}
f(\sigma) \in \mathcal{C}(f(P), \alpha) \Leftrightarrow \operatorname{Rad}(f(\sigma)) \leq \alpha \Rightarrow \operatorname{Rad}(\sigma) \leq \alpha+\varepsilon \\
\Leftrightarrow \operatorname{Rad}(\sigma) \leq \alpha \Leftrightarrow \sigma \in \mathcal{C}(P, \alpha),
\end{array}
$$

yielding $(i i)$. Consider a graph $G$ whose flag complex is an $(\alpha, \beta)$ almost complex and let $p$ and $q$ be two points of $P$ such that $\| f(p)-$ $f(q) \| \leq 2 \alpha$. We have $\|p-q\| \leq 2 \alpha+2 \varepsilon$ and therefore using condition (5) $\|p-q\| \leq 2 \alpha$. It follows that the edge $\{p, q\}$ belongs to $G$ and consequently the edge $\{f(p), f(q)\}$ belongs to $f(G)$. Similarly, suppose $\|f(p)-f(q)\|>2 \beta^{\prime}$. This implies that $\|p-q\|>2 \beta^{\prime}-2 \varepsilon>2 \beta$ by condition (4) and therefore the edge $\{p, q\}$ does not belong to $G$. Hence, the edge $\{f(p), f(q)\}$ does not belong to $f(G)$, showing $(i i i)$. 